\title{
Proposed sets of critical exponents for randomly branched polymers, using a known
} string theory model

\author{
N.H. March ${ }^{1,2,3}$ and A.J. Moreno ${ }^{1,4, *}$ \\ ${ }^{1}$ Donostia International Physics Center, \\ Paseo Manuel de Lardizabal 4, E-20018 San Sebastián, Spain \\ ${ }^{2}$ Department of Physics, University of Antwerp, Antwerp, Belgium; \\ ${ }^{3}$ Oxford University, Oxford, United Kingdom \\ ${ }^{4}$ Centro de Física de Materiales (CSIC-UPV/EHU) and Materials Physics Center; \\ Paseo Manuel de Lardizabal 5, E-20018 San Sebastián, Spain \\ *E-mail address: wabmosea@ehu.es
}

\begin{abstract}
The critical exponent $v$ for randomly branched polymers with dimensionality $d$ equal to three, is known exactly as $1 / 2$. Here, we invoke an already available string theory model to predict the remaining static critical exponents. Utilizing results of Hsu et al., (Comput. Phys. Commun. 169 (2005) 114), results are added for $d=8$. Experiment plus simulation would now be important to confirm, or if necessary to refine, the proposed values.
\end{abstract}

Key words: critical exponent, branched polymers, lattice animals. 
Scaling properties of macromolecular objects have motivated a large body of research over past decades. In the original approach by Flory, the size of a linear polymer chain in good solvent (swollen chain) was estimated by adding an excluded volume term to the entropic contribution of the free energy of a Gaussian chain [1]. In this approach, the size of the swollen chain depends on the polymerization degree $N$ as $\mathrm{R} \sim N^{v}$, with $v=3 / 5$. In the case of a $\theta$-solvent or a polymer melt, excluded volume interactions are screened [1], large-scale Gaussian statistics are recovered, and the scaling exponent becomes $v=1 / 2$. Beyond these well-known relations, increasingly complex macromolecular topologies may result in non-trivial scaling behaviour, as in the case of e.g., dendrimers [2] or ring polymers forming crumpled globules in the melt state [3].

In a recent study, Zhang and March [4] pointed out the potential for treating critical exponents of the pioneering work of Iqbal, Liu and Mezei [5] (ILM below). These authors used gravity/conformal field theory duality (or AdS/CFT as often written) to model critical exponents by means of a parameter $v_{\text {ILM. }}$ (see eqs. (1) to (4) below). In [4] it was demonstrated that $v_{\text {ILM }}$ could depend on both universality class $n$ and dimensionality $d$. Below, in the discussion of randomly branched polymers and lattice animals, these belong to the same universality class, so that only $d$ is varied. At this point it is worth mentioning that other families of (non-randomly) branched polymers may belong to other universality classes (e.g., the class of percolation in the case of the branched polymer growth model [6]).

In connection with the topic of scaling behaviour of macromolecular objects, and following the work in [4], the writers have only now become aware of the continuing 
interest in the area of branched polymers. Randomly branched polymers in good solvent can be represented as lattice animals $[7,8,9]$. In particular, the exponent $v$ is known exactly in such assemblies; for dimensionality $d=3$ namely $v=1 / 2$ (see e.g., seminal work by Parisi and Sourlas [9]). This is unique knowledge for a critical exponent in three dimensions from first principles theory.

Below, therefore, we set out to predict the remaining five static critical exponents for randomly branched polymers by using the above exact value $v=1 / 2$ in conjunction with the ILM model, which is characterized by a single parameter denoted below by $v_{\text {ILM }}$. Then it was demonstrated in [4] that $v$ is given in terms of the dimensionality $d$ and $v_{\text {ILM }}$ as

$$
v=\frac{1}{2 \mathrm{~d} v_{I L M}}
$$

For randomly branched polymers, we set $d=3$ and $v=1 / 2$ in eqn (1) to find the model parameter $v_{\text {ILM }}$ as $1 / 3$.

We now invoke immediately the results of ILM [5] that [see also eqns (1)-(3) in [4]]:

$$
\begin{gathered}
\alpha=2-\frac{1}{2 v_{\mathrm{ILM}}} \\
\beta=\frac{\frac{1}{2}-v_{\mathrm{ILM}}}{2 v_{\mathrm{ILM}}}
\end{gathered}
$$

and

$$
\delta=\frac{\frac{1}{2}+v_{\mathrm{ILM}}}{\frac{1}{2}-v_{\mathrm{ILM}}}
$$

It must be noted that these scaling relations are appropriate below the lower critical dimension, which is exactly 8 for lattice animals. At the critical dimension there are 
logarithmic corrections, and this is also true for self-avoiding random-walks with attractive interactions at their $\theta$-point, where the critical dimension is 3. [8,9].

Using the value $v_{\mathrm{ILM}}=1 / 3$ already obtained above, insertion in eqns (2)-(4) gives the predictions for $\alpha, \beta$ and $\delta$ recorded in Table 1 below. For the known relation between $\gamma / v$ and $\eta$, namely [see e.g., [7]]

$$
\gamma / v=(2-\eta)
$$

we invoke $\gamma / v=2$ from Table 1 to find $\eta=0$, which we have also entered there. The final static exponent $\delta$ is obtained from the relation, which goes back at least to Hubbard and Schofield [10] (see also [4]):

$$
\delta=\frac{\mathrm{d}+(2-\eta)}{\mathrm{d}-(2-\eta)}
$$

By putting $\eta=0$ from Table 1 , plus dimensionality $d=3$, we find $\delta=5$, which is again recorded in Table 1 . The same $\delta$, of course, follows also from eq. (4) when $v_{\text {ILM }}=1 / 3$ is inserted.

In summary, the main results of this present article are contained in Tables 1 and 2. The values of $\alpha, \beta, \gamma, \delta$ and $\eta$ recorded in Table 1 are based on the exactly known value $v=$ $1 / 2$ for $d=3$, plus the ILM string theory model with the parameter $v_{\mathrm{ILM}}=1 / 3$ obtained above. In Table 2 we then show the values of $v_{\mathrm{ILM}}, \alpha, \beta, \gamma, \delta$, also for $d=3$ and 8 . These are obtained, through eqs. (1-4), by using the exact values of the critical exponent $v$ for these dimensionalities (see Hsu et al., Table 1 in Ref. [8]). As to future directions, simulation on any of the other five exponents predicted here would, of course, be important in either confirming, or otherwise refining, the results proposed in this article. 


\section{Acknowledgements}

N.H.M. wishes to acknowledge that his contribution to this study was brought to fruition during a visit to DIPC in 2015. He thanks Professor P.M. Echenique for making this visit possible and for most generous hospitality. Both authors wish to thank Professor J. Colmenero for proposing this collaboration during N.H.M.'s visit.

References:

[1] M. Rubinstein and R.H. Colby, Polymer Physics, (Oxford University Press: New York, 2003)

[2] M. Ballauff and C.N. Likos, Angew. Chem. Int. Ed. 43 (2004) 2998.

[3] J.D. Halverson, W.B. Lee, G.S. Grest, A.Y. Grosberg, and K. Kremer, J. Chem. Phys. 134 (2011) 204904.

[4] Z.D. Zhang, N.H. March, Phys. Chem. Liquids 51 (2013) 261.

[5] N. Iqbal, H. Liu and M. Mezei, in String Theory and its Applications, Editors M. Dine,

T. Banks and S. Sachdev, World Scientific, Singapore, (2012) page 707, chapter 13.

[6] A. Bunde, S. Havlin, M. Porto, Phys. Rev. Lett. 74 (1995) 2714.

[7] J. Cardy, Scaling and Renormalization in Statistical Physics, (Cambridge University Press, UK, 1996).

[8] H.-P. Hsu, W. Nadler and P. Grassberger, Comput. Phys. Commun. 169 (2005) 114.

[9] G. Parisi and N. Sourlas, Phys. Rev. Lett. 46 (1981) 871.

[10] J. Hubbard, P. Schofield, Phys. Lett. A 40 (1972) 245. 
Table 1 Proposed critical exponents for randomly branched polymers, with $d=3$, obtained by combining the known exact value $v=1 / 2$ with the ILM model, with parameter derived here: namely $v_{\mathrm{ILM}}=1 / 3$.

\begin{tabular}{|l|l|l|l|l|l|}
\hline$\alpha$ & $\beta$ & $\gamma$ & $\delta$ & $\eta$ & $\nu$ \\
\hline $1 / 2$ & $1 / 4$ & 1 & 5 & 0 & $1 / 2$ \\
\hline
\end{tabular}

Footnote to Table. Note that $\alpha+2 \beta+\gamma=2$, which is Rushbrooke's relation.

Table 2 String theory parameter $v_{\text {ILM }}$, obtained by using results of Hsu et al. (from Table I in Ref. [8]). The corresponding exponents $\alpha, \beta, \delta$ are included.

\begin{tabular}{|l|l|l|}
\hline Dimensionality d & 3 & 8 \\
\hline Critical exponent $v$, (Hsu et al. [7]) & $1 / 2$ & $1 / 4$ \\
\hline String theory parameter $v_{\text {ILM }}$ in eq. (1) & $1 / 3$ & $1 / 4$ \\
\hline$\alpha$ from eq. (2) & $1 / 2$ & 0 \\
\hline$\beta$ from eq. (3) & $1 / 4$ & $1 / 2$ \\
\hline$\delta$ from eq. (4) & 5 & 3 \\
\hline
\end{tabular}

Footnote to Table. Note that, from Parisi and Sourlas [9], the exponent $\theta$ discussed by Hsu et al. [8] is related to $v$ by $\theta=(d-2) v+1$. For $d=8, \theta=6 v+1=5 / 2$. 